\title{
Local control matters
}

\author{
Ronald B. Levitin, Daniel A. Hamstra
}

Department of Radiation Oncology, Oakland University William Beaumont School of Medicine, Dearborn, MI, USA

Correspondence to: Daniel A. Hamstra, MD, PhD. Department of Radiation Oncology, Oakland University William Beaumont School of Medicine, 18101 Oakwood Boulevard, Dearborn, MI 48124, USA. Email: Daniel.Hamstra@Beaumont.org.

Provenance and Peer review: This article was commissioned by the editorial office, Translational Andrology and Urology. The article did not undergo external peer review.

Comment on: Kishan AU, Chu FI, King CR, et al. Local Failure and Survival After Definitive Radiotherapy for Aggressive Prostate Cancer: An Individual Patient-level Meta-analysis of Six Randomized Trials. Eur Urol 2020;77:201-8.

Submitted Apr 29, 2020. Accepted for publication May 11, 2020.

doi: 10.21037/tau-2020-06

View this article at: http://dx.doi.org/10.21037/tau-2020-06

Men with high-risk localized prostate cancer (PC) represent an important patient population for whom treatment decisions are complex. Compared to the average patient they have greater risk of distant metastasis (DM) and eventual PC-specific mortality (PCSM). To some this raises the question of the impact of local recurrence in the natural history of the disease. A growing body of evidence clearly demonstrates that local control in PC improves DM-free survival (DMFS) and overall survival (OS).

The recent publication by Kishan et al., examined the link between local control and DMFS, PCSM, and OS in high-risk patients (1). They utilized patient-level data encompassing 992 patients with high-risk PC, by virtue of Gleason Grade Groups 4 or 5 (Gleason scores of 8-10), from six randomized trials of external beam radiation therapy (EBRT). Four of the six trials relied on palpable findings to determine local failure (LF). With a median follow-up of 6.4 years, they demonstrated a significant association of LF with OS [hazard ratio (HR) 1.7; 95\% confidence interval (CI): 1.37-2.10], PCSM (HR 3.1; 95\% CI: 2.33-4.12) and DM (HR 1.9; 95\% CI: 1.54-2.39). Patients without LF had a significantly decreased risk of death compared to those with LF (HR 0.13; 95\% CI: 0.04-0.41) while those with LF had a significantly increased risk of metastasis or death (HR 2.46; 95\% CI: 1.22-4.93). Furthermore, they utilized Markov proportional hazard models to quantitatively estimate the relative contributions of transitions between each disease state (disease free, LF, $\mathrm{DM}$, or death) on each outcome. As the underlying trials from which the patients data were extracted were analyzing the role of androgen deprivation therapy (ADT), the authors were also able to further investigate the influence of $\mathrm{ADT}$ duration on these outcomes and found that long-term and lifelong $\mathrm{ADT}$ had a significant impact on the transition to LF (HR 0.34; 95\% CI: $0.20-0.59$ and HR 0.17; 95\% CI: 0.06-0.49 for long-term and lifelong compared to no ADT respectively). This analysis supplements a trend in the literature demonstrating that the higher the risk-group the greater the benefit to local therapy.

The benefit of local control for PC was first demonstrated by the SPCG-4 trial which randomized patients to watchful waiting or radical prostatectomy (RP). With extended follow-up at 18 years there was a benefit to local therapy in the rate of DM (38\% vs. 26\%), PCSM (29\% vs. $18 \%)$ and overall mortality (OM) (69\% vs. 56\%) (2). Notably, although this trial was intended to represent a lowrisk population, it was performed in an era before prostatespecific antigen (PSA) screening, with $88 \%$ having palpable disease. As such this should not be taken to represent today's low-risk, active surveillance population.

For locally advanced disease, three randomized trials of ADT with or without EBRT also demonstrated the benefit of local therapy (3-5). SPCG-7 had the longest follow-up and demonstrated a clear benefit to the addition of EBRT on PCSM (HR 0.42; 95\% CI: 0.31-0.57) and OM (HR 0.70; $95 \%$ CI: $0.58-0.84)$ with a $17 \%$ reduction in death from $\mathrm{PC}$ at 15 -years (34\% vs. $17 \%$ ) and $10 \%$ improvement in OM (61\% vs. 51\%). The UK/NCIC PR.3 trial had nearly identical estimates for the benefit in OM (HR 0.70; $95 \%$ CI: $0.57-0.85)$ and PCSM (0.46; 95\% CI: 0.34-0.61). The 
French TAP-32 trial had the shortest follow-up and a sample size powered only for the primary endpoint of biochemical failure (BF), not for PCSM or OS (5). Despite a lack of difference in more concrete endpoints the TAP-32 trial did show a significant improvement in PFS (HR 0.27; 95\% CI: $0.17-0.39)$. As such these trials clearly demonstrate that local therapy, in this case EBRT, can decrease metastasis and improve both prostate cancer specific survival (PCSS) and $\mathrm{OS}$ in patients with higher-risk PC treated with ADT.

Post-operative EBRT after RP with high-risk features can also be viewed as a form of local intensification. Of the three classic adjuvant EBRT trials, the SWOG 8794 trial had the highest-risk patients. A third of patients had a postoperative PSA $>0.2 \mathrm{ng} / \mathrm{mL}$ prior to EBRT, and yet even with a relatively modest dose of 60-64 Gy, they were able to demonstrate significant improvements in DMFS (HR 0.71; 95\% CI: 0.54-0.94) and OS (HR 0.72; 95\% CI: 0.55-0.96) $(6,7)$. The EORTC 22911 trial similarly demonstrated a benefit to $\mathrm{BF}$, and 10 year loco-regional failure with adjuvant RT. This trial had $\sim 30 \%$ of patients having PSA $>0.2 \mathrm{ng} / \mathrm{mL}$. In contrast, the ARO 96-02 trial had the lowest-risk patients and did not show a benefit in OS although adjuvant radiation therapy (RT) substantially reduced $\mathrm{BF}$. The latest wave of post-operative trials, RADICALS-RT, RAVES, and the GETUG-AFU 17 have undergone meta-analysis of their first reports by the ARTISTIC collaboration. These data were presented at ESMO 2019 and indicate that early salvage is a reasonable alternative to adjuvant therapy for most men, suggesting that the benefit seen in the early trials is derived from intensified treatment of persistent local disease.

More importantly there may even be a benefit of EBRT to the prostate in men with early metastatic PC. In the STAMPEDE trial (8), patients with metastatic PC were treated with standard of care systemic therapy with or without prostate EBRT. The addition of EBRT in all patients improved failure free survival (FFS, HR 0.76; $95 \%$ CI: $0.68-0.84$ ) but did not improve OS (HR 0.92; $95 \%$ CI: $0.80-1.06)$ ). However, when analyzed in pre-specified subgroups, patients with low metastatic disease burden were found to have benefit in terms of OS (HR 0.68; 95\% CI: 0.52-0.92), PCSM (HR 0.65; 95\% CI: 0.47-0.90) and FFS (HR 0.59; 95\% CI: 0.49-0.72) with a 7\% improvement in prostate-cancer specific survival at 3-years (86\% vs. $79 \%$ ). High metastatic burden was defined, as in previous trials, as four or more bone metastases with at least one outside the axial skeleton and/or visceral metastases, and there was no difference in any of outcomes with RT added in patients with a high disease burden, suggesting that there is a potential upper limit to those who benefit from local therapy. In a separate STAMPEDE report establishing the benefit of Abiraterone in the ADT sensitive status (9), the subgroup analysis for FFS demonstrates a significant interaction for an increased benefit with planned EBRT $(\mathrm{P}=0.02)$ and borderline significant interaction with nonmetastatic patients $(\mathrm{P}=0.08)$. That these two subgroups may have more of a benefit from Abiraterone potentially suggest that it contributes to the local effect of EBRT just as conventional $\mathrm{ADT}$ was previously demonstrated to improve local control.

As technology has improved, EBRT studies sought to identify more direct evidence of a benefit to local intensification through dose-escalation. Six randomized trials (10-15) of over 4,000 patients assessed conventionally fractionated dose-escalation and uniformly succeeded in demonstrating a dose-response effect for BF, provided evidence that LF portends a worse prognosis, and in some cases confirmed that LF may lead to a late wave of metastatic disease. The relationship between LF and DM has further gained importance as DM has recently been validated as a surrogate for PCSS by the ICECaP working group (16). The relationship between LF and DM has been directly explored in several retrospective series. Investigators from Massachusetts General Hospital noted that the hazard rate for distant failure was initially high for all patients, but in those with LF it increased with time (17). This prompted the hypothesis that early metastases represent micro-metastatic disease at the time of diagnosis that emerge after treatment, whereas subsequent LF results in a "late wave" of metastases. This was further supported by a series at Fox Chase Cancer Center while emphasizing the inverse relationship between EBRT dose and distant failure with higher RT doses appearing to decrease late distant metastases (18). These early reports relied on palpable recurrence to determine LF while investigators from Memorial Sloan Kettering included only biopsy-proven LF. In their series, post-treatment biopsy status was the strongest predictor for DM [HR 2.39; 95\% CI: not reported (NR)] and DSS (HR 3.3; 95\% CI: NR) (19). They also noted a dose-response effect on LF. More recently, a secondary analysis of the RTOG 9408 trial demonstrated that biopsy-proven LF is a strong predictor of DM (HR 2.4; 95\% CI: 1.3-4.4), DSS (HR 3.8; 95\% CI: 1.9-7.5) and OS (HR 1.23; 95\% CI: 0.95-1.6) (20). In RTOG 9408, the population favored low- and intermediate-risk patients, and an important caveat noted that these findings may not 
be generalizable to higher-risk patients such as those with higher Gleason scores.

The work by Kishan et al., then takes this further by pooling outcomes from randomized trials of prostate EBRT and assessed the impact of LF on DM, PCSS, and OS specifically in high-risk patients. Notably it is likely that none of these individual trials had sufficient sample size to demonstrate that LF [based upon digital rectal exam (DRE)] correlated with these clinical endpoints. However, in the pooled analysis the impact of LF became evident (1). When limited to those with pathologically the most aggressive PCs_-Grade Group 4 and Grade Group 5 at biopsy—-there was a substantial adverse impact of LF on DMFS (HR 1.9; 95\% CI: 1.54-2.39), PCSS (HR 3.1; 95\% CI: 2.33-4.12) and even OS (HR 1.7; 95\% CI: 1.37-2.10). These estimates correlate well with the results previously observed for biopsy proven LF from MSKCC and RTOG 9408.

Does this really mean that RT is needed in these most aggressive patients? The alternative theory argues that LF represents inherently more aggressive biology that portends both increased DM and LF. In a single institutional retrospective series of patients treated with dose-escalated EBRT (21), the presence of any Gleason pattern 5 in those with Gleason 8-10 PC as compared to those without pattern 5 , predicted significantly worse outcomes for all endpoints, including time to BF, DMFS (HR 3.4; 95\% CI: 1.7-7.1), PCSS (HR 12.9; 95\% CI: 5.4-31) and OS (HR 3.6; 95\% CI: 2.0-6.5). This finding was also borne out in a population-based SEER analysis (22). Interestingly, in a secondary analysis of two cooperative group trials, RTOG 9202 and RTOG 9902, Gleason pattern 5 disease resulted in increased distant metastases but not LF (23). This recognition of a more aggressive phenotype has made its way into the newer Gleason Grade Groups, as well as the National Comprehensive Cancer Network (NCCN) riskstratification where the highest Gleason scores represent very-high risk disease. As a result, some have argued that LF is a sign of aggressive disease and not necessarily the cause of metastasis.

The Markov models utilized by Kishan $e t$ al., attempt to tease out this interaction and illustrate the relative impact of de novo DM and DM following an initial LF. Accounting for the impact of ADT duration, they note that in the first 5 years, as much as $81-96 \%$ of failures are directly transitioning from recurrence-free to metastatic, suggestive of a significant proportion of patients with occult metastatic disease at diagnosis which manifest after treatment. In contrast, after 5 years of follow-up, the proportion of
DM that present following an initial LF increases to approximately $50 \%$ in those treated without or with a short duration $\mathrm{ADT}$ but not in those treated with long-term or lifelong ADT, suggesting that without adequate ADT LF can become a significant contributor to DM (1). These data strongly support that local control dose matter even in those with the highest pathologic risk disease.

One logical way to increase radiation dose to the prostate and improve local control is through the use of brachytherapy (BT). Three randomized trials have explored dose-escalation through the combination of BT and EBRT. The first was a single-center study from Ontario, performed in the era before 3D-based treatment planning with conventional fractionation (24). This is the only study reporting long-term outcomes, with a median follow up of 14 years, and found significant improvement in biochemical progression free survival (bPFS) (HR 0.53; 95\% CI: $0.31-$ 0.88 ) but not DMFS, PCSM, or OM. The next singleinstitution randomized trial utilized more modern technique but has only reported 7-year results (25). They also found a significant improvement in median bPFS (116 vs. 74 months, $\mathrm{P}=0.04$ ), but no difference in OS. The Canadian multicenter ASCENDE-RT utilized 12 months of ADT for all and randomized between a low-dose rate (LDR) BT or dose-escalated EBRT of 78 Gy (26). Biochemical PFS was significantly inferior in the control arm (HR 2.04; 95\% CI: 1.25-3.33) but no significant differences in DMFS, PCSS, or OS were noted. All three trials enrolled intermediate and high-risk patients, and all found significant improvements in biochemical and clinical relapse-free survival with the addition of a BT boost. Nevertheless, none have thus far demonstrated an OS benefit, although only the earliest trial has published long-term results.

Just as the current study by Kishan et al. focused upon LF in those with the highest Gleason scores, the addition of BT boost to improve local control has shown promising results in a retrospective multi-institutional study (27). Collecting data from 12 centers and focusing on those with Gleason scores of 9-10 allowed for a comparison of RP, EBRT and EBRT + BT with an enriched event rate. After propensity score adjustment they reported a significant improvement in DMFS with BT boost (HR 0.27; 95\% CI: 0.17-0.43 and HR 0.30; 95\% CI: $0.19-0.47$ for EBRT + BT vs. RP and vs. EBRT alone, respectively) and PCSM (HR 0.38; $95 \%$ CI: $0.19-0.73$, and HR 0.36; 95\% CI: 0.18-0.70). Tilki et al. followed this analysis with one of their own and noted that $\mathrm{EBRT}+\mathrm{ADT}+\mathrm{BT}$ is the most intensive RT that can be offered ("MaxRT"), while RP can be supplemented 
with adjuvant RT, ADT, or both ("MaxRP"). In their multi-institutional retrospective analysis both of these "Max Therapies" yielded similar risk for PCSM and allcause mortality (28) with each showing improvements with intensified local therapy compared to less aggressive RT or surgical approaches. A recent systematic review supports these findings showing EBRT alone to be inferior to RP, with this difference mitigated with the addition of ADT. While MaxRT was superior to RP and MaxRP was comparable to MaxRT (29). These analyses, although constituting weaker evidence than a randomized trial, point to a benefit to further intensification of local therapy.

While Grade Groups 4 and 5 portend a poorer prognosis, there may be additional patients at higher risk for whom local intensification may play a role. Several investigators have attempted to risk stratify patients with the use of genomic classifiers in the adjuvant setting. Den et al. reported in a retrospective two-center cohort that a genomic classifier was able to better classify patients according to their risk of DM compared to the best available contemporary nomograms in the post-operative setting. Furthermore, they were able to demonstrate that in patients classified as average or high-risk, an adjuvant approach decreased rates of DM significantly compared to a salvage radiotherapy approach (30).

In conclusion, for PC local control matters and it appears to matter most in those with the highest-risk disease up to and including even those with low-volume metastatic disease. How best to optimize local control remains to be seen. Randomized trials of ADT provide the strongest data with the highest level of evidence while dose escalation with a BT boost or the addition of post-operative RT for early salvage are also important strategies, but with weaker evidence. Importantly, these data further strengthen the argument that we should not give up on those with the highest-risk disease. Despite the substantially higher risk of DM in Grade Group 4-5 disease, this does not mean that local control does not play a role. Randomized trials of newer agents and improved diagnostics for risk stratification, such as more accurate imaging and genomic classifiers, may also help us to identify those patients who benefit from intensified local therapy. Taken together, there is a compelling case that just as active surveillance is most appropriate in those with low-risk PC for whom local therapy is unlikely to change OS, treatment with radiotherapy and potentially even intensified RT for local control in higher risk patients appears to be more relevant than previously appreciated.

\section{Acknowledgments}

Funding: None.

\section{Footnote}

Conflicts of Interest: Both authors have completed the ICMJE uniform disclosure form (available at http://dx.doi. org/10.21037/tau-2020-06). DAH reports grants and personal fees from Augmenix, personal fees from Boston Scientific, outside the submitted work. RBL author has no conflicts of interest to declare.

Ethical Statement: The authors are accountable for all aspects of the work in ensuring that questions related to the accuracy or integrity of any part of the work are appropriately investigated and resolved.

Open Access Statement: This is an Open Access article distributed in accordance with the Creative Commons Attribution-NonCommercial-NoDerivs 4.0 International License (CC BY-NC-ND 4.0), which permits the noncommercial replication and distribution of the article with the strict proviso that no changes or edits are made and the original work is properly cited (including links to both the formal publication through the relevant DOI and the license). See: https://creativecommons.org/licenses/by-nc-nd/4.0/.

\section{References}

1. Kishan AU, Chu FI, King CR, et al. Local Failure and Survival After Definitive Radiotherapy for Aggressive Prostate Cancer: An Individual Patient-level Meta-analysis of Six Randomized Trials. Eur Urol 2020;77:201-8.

2. Bill-Axelson A, Holmberg L, Garmo H, et al. Radical prostatectomy or watchful waiting in early prostate cancer. N Engl J Med 2014;370:932-42.

3. Fosså SD, Wiklund F, Klepp O, et al. Ten- and 15yr Prostate Cancer-specific Mortality in Patients with Nonmetastatic Locally Advanced or Aggressive Intermediate Prostate Cancer, Randomized to Lifelong Endocrine Treatment Alone or Combined with Radiotherapy: Final Results of The Scandinavian Prostate Cancer Group-7. Eur Urol 2016;70:684-91.

4. Mason MD, Parulekar WR, Sydes MR, et al. Final Report of the Intergroup Randomized Study of Combined Androgen-Deprivation Therapy Plus Radiotherapy Versus Androgen-Deprivation Therapy Alone in Locally 
Advanced Prostate Cancer. J Clin Oncol 2015;33:2143-50.

5. Sargos P, Mottet N, Bellera C, et al. Long-term androgen deprivation, with or without radiotherapy, in locally advanced prostate cancer: updated results from a phase III randomised trial. BJU Int 2019;125:810-6.

6. Swanson GP, Hussey MA, Tangen CM, et al. Predominant Treatment Failure in Postprostatectomy Patients Is Local: Analysis of Patterns of Treatment Failure in SWOG 8794. J Clin Oncol 2007;25:2225-9.

7. Thompson IM, Tangen CM, Paradelo J, et al. Adjuvant radiotherapy for pathological T3N0M0 prostate cancer significantly reduces risk of metastases and improves survival: long-term followup of a randomized clinical trial. J Urol 2009;181:956-62.

8. Parker CC, James ND, Brawley CD, et al. Radiotherapy to the primary tumour for newly diagnosed, metastatic prostate cancer (STAMPEDE): a randomised controlled phase 3 trial. Lancet 2018;392:2353-66.

9. James ND, de Bono JS, Spears MR, et al. Abiraterone for Prostate Cancer Not Previously Treated with Hormone Therapy. N Engl J Med 2017;377:338-51.

10. Kuban DA, Tucker SL, Dong L, et al. Long-term results of the M. D. Anderson randomized dose-escalation trial for prostate cancer. Int J Radiat Oncol Biol Phys 2008;70:67-74.

11. Al-Mamgani A, van Putten WL, Heemsbergen WD, et al. Update of Dutch multicenter dose-escalation trial of radiotherapy for localized prostate cancer. Int J Radiat Oncol Biol Phys 2008;72:980-8.

12. Zietman AL, Bae K, Slater JD, et al. Randomized trial comparing conventional-dose with high-dose conformal radiation therapy in early-stage adenocarcinoma of the prostate: long-term results from proton radiation oncology group/american college of radiology 95-09. J Clin Oncol 2010;28:1106-11.

13. Beckendorf V, Guerif S, Prisé EL, et al. 70 Gy Versus 80 Gy in Localized Prostate Cancer: 5-Year Results of GETUG 06 Randomized Trial. Int J Radiat Oncol Biol Phys 2011;80:1056-63.

14. Dearnaley DP, Jovic G, Syndikus I, et al. Escalateddose versus control-dose conformal radiotherapy for prostate cancer: long-term results from the MRC RT01 randomised controlled trial. Lancet Oncol 2014;15:464-73.

15. Michalski JM, Moughan J, Purdy J, et al. Effect of Standard vs Dose-Escalated Radiation Therapy for Patients With Intermediate-Risk Prostate Cancer: The NRG Oncology RTOG 0126 Randomized Clinical Trial.
JAMA Oncol 2018;4:e180039.

16. Xie W, Regan MM, Buyse M, et al. Metastasis-Free Survival Is a Strong Surrogate of Overall Survival in Localized Prostate Cancer. J Clin Oncol 2017;35:3097-104.

17. Coen JJ, Zietman AL, Thakral H, et al. Radical Radiation for Localized Prostate Cancer: Local Persistence of Disease Results in a Late Wave of Metastases. J Clin Oncol 2002;20:3199-205.

18. Jacob R, Hanlon AL, Horwitz EM, et al. The relationship of increasing radiotherapy dose to reduced distant metastases and mortality in men with prostate cancer. Cancer 2004;100:538-43.

19. Zelefsky MJ, Reuter VE, Fuks Z, et al. Influence of Local Tumor Control on Distant Metastases and Cancer Related Mortality After External Beam Radiotherapy for Prostate Cancer. J Urol 2008;179:1368-73.

20. Krauss DJ, Hu C, Bahary JP, et al. Importance of Local Control in Early-Stage Prostate Cancer: Outcomes of Patients With Positive Post-Radiation Therapy Biopsy Results Treated in RTOG 9408. Int J Radiat Oncol Biol Phys 2015;92:863-73.

21. Sabolch A, Feng FY, Daignault-Newton S, et al. Gleason Pattern 5 Is the Greatest Risk Factor for Clinical Failure and Death From Prostate Cancer After Dose-Escalated Radiation Therapy and Hormonal Ablation. Int $\mathrm{J}$ Radiat Oncol Biol Phys 2011;81:e351-60.

22. Rusthoven CG, Carlson JA, Waxweiler TV, et al. The prognostic significance of Gleason scores in metastatic prostate cancer. Urol Oncol 2014;32:707-13.

23. Hamstra DA, Pugh SL, Lepor H, et al. Gleason pattern 5 is associated with an increased risk for metastasis following androgen deprivation therapy and radiation: An analysis of RTOG 9202 and 9902. Radiother Oncol 2019;141:137-43.

24. Dayes IS, Parpia S, Gilbert J, et al. Long-Term Results of a Randomized Trial Comparing Iridium Implant Plus External Beam Radiation Therapy With External Beam Radiation Therapy Alone in Node-Negative Locally Advanced Cancer of the Prostate. Int J Radiat Oncol Biol Phys 2017;99:90-3.

25. Hoskin PJ, Rojas AM, Bownes PJ, et al. Randomised trial of external beam radiotherapy alone or combined with high-dose-rate brachytherapy boost for localised prostate cancer. Radiother Oncol 2012;103:217-22.

26. Morris WJ, Tyldesley S, Rodda S, et al. Androgen Suppression Combined with Elective Nodal and Dose Escalated Radiation Therapy (the ASCENDE-RT Trial): An Analysis of Survival Endpoints for a Randomized Trial Comparing a Low-Dose-Rate Brachytherapy Boost to 
a Dose-Escalated External Beam Boost for High- and Intermediate-risk Prostate Cancer. Int J Radiat Oncol Biol Phys 2017;98:275-85.

27. Kishan AU, Cook RR, Ciezki JP, et al. Radical Prostatectomy, External Beam Radiotherapy, or External Beam Radiotherapy With Brachytherapy Boost and Disease Progression and Mortality in Patients With Gleason Score 9-10 Prostate Cancer. JAMA 2018;319:896-905.

28. Tilki D, Chen MH, Wu J, et al. Surgery vs Radiotherapy in the Management of Biopsy Gleason Score 9-10 Prostate Cancer and the Risk of Mortality. JAMA Oncol

Cite this article as: Levitin RB, Hamstra DA. Local control matters. Transl Androl Urol 2020;9(3):991-996. doi:10.21037/tau2020-06
2019;5:213-20.

29. Greenberger BA, Zaorsky NG, Den RB. Comparison of Radical Prostatectomy Versus Radiation and Androgen Deprivation Therapy Strategies as Primary Treatment for High-risk Localized Prostate Cancer: A Systematic Review and Meta-analysis. Eur Urol Focus 2020;6:404-18.

30. Den RB, Yousefi K, Trabulsi EJ, et al. Genomic classifier identifies men with adverse pathology after radical prostatectomy who benefit from adjuvant radiation therapy. J Clin Oncol 2015;33:944-51. Erratum in: Errata. J Clin Oncol 2015;33:1416. 